\title{
Effects of transcutaneous electrical acupoint stimulation on perioperative immune function and postoperative analgesia in patients undergoing radical mastectomy: A randomized controlled trial
}

\author{
$\mathrm{LI} \mathrm{AO}^{1 *}, \mathrm{JINLIN} \mathrm{SHI}^{2 *}$, YAOWU BAI $^{1}$, SHAN ZHANG $^{3}$ and JIANHUI GAN ${ }^{2}$ \\ ${ }^{1}$ Department of Anesthesiology, Tangshan Maternity and Child Healthcare Hospital; \\ ${ }^{2}$ Department of Anesthesiology, Tangshan People's Hospital and Tangshan Cancer Hospital, \\ North China University of Science and Technology, Tangshan, Hebei 063000; ${ }^{3}$ Department of Anesthesiology, \\ Second Hospital of Hebei Medical University, Shijiazhuang, Hebei 050000, P.R. China
}

Received February 29, 2020; Accepted November 26, 2020

DOI: $10.3892 /$ etm.2021.9615

\begin{abstract}
Radical mastectomy may lead to suppression of cellular immune function in patients with malignant tumors. Transcutaneous electrical acupoint stimulation (TEAS) is widely used in clinical practice. However, there have been relatively few studies on the effects of TEAS on postoperative analgesia and immune function. The present study aimed to evaluate the effects of TAES on postoperative pain and immune function in patients undergoing radical mastectomy. A total of 65 patients were enrolled and allocated to either receive TEAS or sham TEAS. TEAS was implemented on bilateral Hegu (LI4), Neiguan (PC6) and Zusanli (ST36) acupoints simultaneously for $30 \mathrm{~min}$ before induction of anesthesia at 4 and $12 \mathrm{~h}$ post-operation. The primary outcomes included visual analogue scale (VAS) scores at $4 \mathrm{~h}\left(\mathrm{~T}_{1}\right), 12 \mathrm{~h} \mathrm{~T}_{2}$ ), $24 \mathrm{~h}\left(\mathrm{~T}_{3}\right)$ and $48 \mathrm{~h}\left(\mathrm{~T}_{4}\right)$ post-operation, and serum levels of IL-2, IL-4, IFN- $\gamma$ and the IL-2/IL-4 ratio at $30 \mathrm{~min}$ before TEAS $\left(\mathrm{T}_{0}\right), \mathrm{T}_{1}, \mathrm{~T}_{2}, \mathrm{~T}_{3}$ and $\mathrm{T}_{4}$. Secondary outcomes included the cumulative time of rescue analgesia within $48 \mathrm{~h}$ post-surgery, as well as the incidence of postoperative nausea and vomiting (PONV) and pruritus. Compared with the sham TEAS group,
\end{abstract}

Correspondence to: Dr Jianhui Gan, Department of Anesthesiology, Tangshan People's Hospital and Tangshan Cancer Hospital, North China University of Science and Technology, 65 Shengli Road, Tangshan, Hebei 063000, P.R. China

E-mail: ganjianhui666@163.com

${ }^{*}$ Contributed equally

Abbreviations: $\mathrm{BIS}$, bispectral index; $\mathrm{P}_{\mathrm{ET}} \mathrm{CO}_{2}$, pressure of end-tidal carbon dioxide; PCA, patient-controlled analgesia; TENS, transcutaneous electrical nerve stimulation; VAS, visual analogue scale

Key words: transcutaneous electrical acupoint stimulation, breast cancer, radical mastectomy, postoperative analgesia, immunity postoperative VAS scores at $\mathrm{T}_{2}$ and $\mathrm{T}_{3}$, the total consumption of opioids in the patient-controlled analgesia (PCA) pump, pressing times of the PCA pump and the incidences of PONV and headache were significantly lower in the TEAS group. The serum levels of IFN- $\gamma$ at $\mathrm{T}_{3}$ and $\mathrm{T}_{4}$, and the serum levels of IL-2 and the IL-2/IL-4 ratio at $\mathrm{T}_{2}, \mathrm{~T}_{3}$ and $\mathrm{T}_{4}$ were higher in the TEAS group compared with the sham TEAS group. By contrast, the serum levels of IL-4 were lower at $T_{2}, T_{3}$ and $\mathrm{T}_{4}$ in the TEAS group compared with the sham TEAS group. The results indicated that TEAS could improve postoperative analgesia, reduce postoperative consumption of opioids and alleviate postoperative side effects. Simultaneously, TEAS was able to reverse decreased serum levels of IL- 2 and IFN- $\gamma$, reduce the level of IL-4 and restore the balance of Th1/Th2, thereby partially attenuating perioperative immune function depression in patients with breast cancer. The current trial was registered prior to participant enrollment at www.chictr.org.cn (Clinical Trial no. ChiCTR1800017768).

\section{Introduction}

Breast cancer is the most frequently diagnosed malignancy and ranks as the leading cause for cancer-related deaths among females worldwide, for which surgery has become the primary treatment strategy (1). Patients with malignant tumors often also display immune dysfunction (2). A number of factors may aggravate immune dysfunction during the perioperative period (3-5). Recently, there has been increasing interest in the influence of perioperative immune function secondary to the surgical stress response, anesthesia and postoperative acute pain (6). Perioperative immune dysfunction may predispose patients to infections, homeostasis disturbances, organ dysfunction and other related complications, including an increased mortality rate $(7,8)$. Therefore, it is crucial to take measures to protect the immune function of patients during the perioperative period.

Postoperative pain may trigger a strong stress response, stimulate the release of inflammatory factors and inhibit 
immune function (9). Therefore, an ideal postoperative analgesic is conducive to the recovery of patients and shortening the length of hospital stay. Currently, opioids providing high analgesic efficacy are commonly used as effective postoperative analgesics (10). However, opioid-induced immunosuppressive effects and adverse reactions including nausea, vomiting, sedation, dizziness and decreased gut motility may delay patient recovery, which limits the application of opioids $(11,12)$.

A recently published meta-analysis suggested that acupuncture is effective in alleviating cancer-related pain, particularly malignancy- and surgery-induced pain (13). Transcutaneous electrical acupoint stimulation (TEAS), as a novel non-invasive acupuncture alternative therapy, combines acupoint stimulation and transcutaneous electrical nerve stimulation (TENS), and displays similarities with traditional acupuncture and moxibustion (14). Due to the advantages of the non-invasive, convenient application and reduced number of side effects of TEAS, it has been increasingly applied in conventional medical settings (15). To date, there have been a few prospective studies comparing the effects of TEAS on postoperative analgesia control and postoperative recovery $(16,17)$.

The concentration of immune cytokines and immunocyte in tumor tissues and plasma is closely related to the cellular immune response to malignant tumors, which may implicate the prognosis of malignant tumors $(18,19)$. Immune cytokines are primarily secreted by $\mathrm{T}$ helper (Th) 1 and Th2 cells, which are associated with the cellular immune responses to malignant tumors. Th1 cells release IFN- $\gamma$, IL-2 and TNF, which are essential for anti-inflammatory and antineoplastic processes $(20,21)$, whereas Th 2 cells secrete proinflammatory cytokines, primarily including IL-4, IL-6 and IL-10 $(19,22)$. Therefore, the release and balance of cytokines during the perioperative period have substantial implications for alterations to immune function (23). The present study investigated the serum levels of IL-2, IFN- $\gamma$ and IL-4, and estimated the balance of Th1/Th2 by calculating the IL-2/IL- 4 ratio to explore whether TEAS could improve immune dysfunction. The present study aimed to identify the effects of TEAS on immune function and postoperative analgesia for patients undergoing radical mastectomy.

\section{Materials and methods}

Participants. The present study was based on the Declaration of Helsinki and the Guidelines on Good Clinical Practice (24). The present study was approved by the Ethics Committee of Human Research of Tangshan People's Hospital (approval no. RMYY-YWLL-2017-1110; Tangshan, China). The trial was also registered prior to participant enrollment at www. chictr.org.cn (clinical trial no. ChiCTR1800017768). Written informed consent was obtained from each patient. Adult female patients (age, 20-65 years) with American Society of Anesthesiologists physical status I or II (25), who were scheduled to undergo elective radical mastectomy of breast cancer in the Department of Anesthesiology, Tangshan People's Hospital and Tangshan Cancer Hospital, North China University of Science and Technology (Tangshan, China) between August 2018 and December 2019, were enrolled in the present study. The exclusion criteria were as follows: i) Body mass index $>30 \mathrm{~kg} / \mathrm{m}^{2}$; ii) severe cardiac or respiratory diseases, significant renal or hepatic impairment or immune disorders; iii) history of immunosuppressive therapy (chemotherapy or radiation) or previous acupuncture/TAES therapies; iv) history of chronic pain, steroid or opioid administration, or alcohol or illegal substance abuse; v) high levels of C-reactive protein or leukocytosis $(>10,000 / \mathrm{ml})$ prior to surgery; and vi) puncture site infection or systemic infection.

Randomization and blinding. A total of 70 patients were enrolled and randomly allocated to the TEAS group or the sham TEAS group using sequentially-numbered sealed envelopes and a random number generator. The allocation ratio between the two groups was 1:1. The envelopes were prepared and distributed by an assistant who was not involved in the present study. An experienced acupuncturist, who was independent of the present study, performed the corresponding interventions. All surgeries were performed by the same group of surgeons. Similarly, a blinded anesthesiologist provided anesthesia implementation and perioperative care. A second anesthesiologist, who was also blinded to the treatment regimen and was not involved in the data analysis, conducted the anesthesia follow-ups.

Study protocol. For patients in both groups, the application of TEAS was implemented at bilateral Hegu (LI-4), Neiguan (PC-6) and Zusanli (ST-36) acupoints simultaneously. The acupoints were identified according to the traditional anatomical location (Fig. 1). LI-4 Hegu is located at the radial side of the dorsum surface of the hand, between the first and second metacarpal bones. ST-36 Zusanli is located at 3 cun below the patella, outside of the anterior crest of the tibia. PC-6 Neiguan is located at 2 cun above the transverse crease of the wrist, between the palmaris longus tendons and the flexor carpi radialis. After acupoint selection and skin disinfection, the electrodes were attached to the target acupoints. A constant electrical stimulation was applied for $30 \mathrm{~min}$ each time with a dense-and-disperse frequency of $2 / 100 \mathrm{~Hz}$ (26) via a HANS LH-202 electrical stimulator (Nanjing Jisheng Medical Technology Co., Ltd.). The optimal intensity was set to mild twitching of the surrounding muscle and individual maximum tolerance (5-10 mA for upper limbs; 10-30 mA for lower limbs) (27,28). The TEAS effect was confirmed by de qi sensation $(27,29)$. Participants in the sham TEAS group underwent electrode attachment on the target acupoints without electronic stimulation.

TEAS was performed for $30 \mathrm{~min}$ prior to the induction of anesthesia. Postoperative TEAS was performed for $30 \mathrm{~min}$ each time at 4 and $12 \mathrm{~h}$ post-surgery on the day of surgery, and administrated three times ( 8 a.m., 2 p.m. and 8 p.m.) daily at postoperative days 1 and 2. Participants in each group were also provided with a patient-controlled analgesia (PCA) pump for postoperative pain control, which was maintained for $48 \mathrm{~h}$ post-surgery. The PCA consisted of $1.5 \mu \mathrm{g} / \mathrm{kg}$ sufentanil (cat. no. 81A09131; Yichang Humanwell Pharmaceutical Co., Ltd.), diluted to $150 \mathrm{ml}$ with normal saline. The basal infusion rate was set to $2 \mathrm{ml} / \mathrm{h}$, with a bolus dose of $0.5 \mathrm{ml}$ and a lockout interval of $15 \mathrm{~min}$.

Standardized anesthesia. Upon arrival in the operating room, all patients were continuously monitored with 
electrocardiography, and the following parameters: Blood pressure, pulse oximetry, pressure of end-tidal carbon dioxide $\left(\mathrm{P}_{\mathrm{ET}} \mathrm{CO}_{2}\right)$ and bispectral index (BIS) (cat. no. MG8001; Sichuan Kehong Medical Equipment Co., Ltd.) were monitored (30). Before anesthesia induction, all patients received midazolam (1-2 mg; cat. no. 20190108; Jiangsu Nhwa Pharmaceutical Co., Ltd.) intravenously. General anesthesia was induced using intravenous sufentanil $(0.3-0.5 \mu \mathrm{g} / \mathrm{kg}$; cat. no. 81A09131; Yichang Humanwell Pharmaceutical Co., Ltd.), propofol (2-3 mg/kg; cat. no. 1809208; Fresenius Kabi) and cisatracurium $(0.2 \mathrm{mg} / \mathrm{kg}$; cat. no. 18082021 ; Jiangsu Hengrui Medicine Co., Ltd.). After intubation, ventilation was adopted with an $8 \mathrm{ml} / \mathrm{kg}$ tidal volume and $\mathrm{P}_{\mathrm{ET}} \mathrm{CO}_{2}$ was maintained at 35-40 mmHg. Propofol and remifentanil (cat. no. 80A06221; Yichang Humanwell Pharmaceutical Co., Ltd.) were titrated to maintain hemodynamic stability intraoperatively by using a target-controlled infusion. During the surgery, the BIS value was maintained at 40-60. Muscle relaxation was facilitated by the intermittent administration of cisatracurium according to surgical requirements. After surgery, muscle relaxation was antagonized with $1 \mathrm{mg}$ neostigmine (cat. no. 1810605; Shanghai Xinyi Pharmaceutical Co., Ltd.) and $0.5 \mathrm{mg}$ atropine (cat. no. 1809131; Tianjin Jinyao Pharmaceutical Co., Ltd.). The patients were sent to the postanesthetic care unit for further monitoring after extubation. The anesthesia time, operation time, volume of blood loss and urine were recorded.

Observation indexes. Visual analogue scale (VAS) scores (31) were recorded quantitatively to assess the postoperative pain intensity at the following time points post-surgery: $4 \mathrm{~h}\left(\mathrm{~T}_{1}\right)$, $12 \mathrm{~h}\left(\mathrm{~T}_{2}\right), 24 \mathrm{~h}\left(\mathrm{~T}_{3}\right)$ and $48 \mathrm{~h}\left(\mathrm{~T}_{4}\right)$. VAS scores ranged from $0-10,0$ indicated no pain and 10 indicated the worst pain. The use of the VAS score was detailed to each patient to ensure the accuracy of the assessment. Subjects were asked to make a mark on the VAS line to indicate the instant pain intensity.

The PCA pump bolus and the total consumption of opioids within $48 \mathrm{~h}$ post-surgery were recorded. In addition, the occurrence of postoperative adverse complications, including postoperative nausea and vomiting (PONV), pruritus, dizziness and headache, were observed up to $48 \mathrm{~h}$ after operation.

Blood sample collection. Peripheral venous blood $(5 \mathrm{ml})$ was drawn from each participant at $30 \mathrm{~min}$ before the first treatment of TEAS $\left(\mathrm{T}_{0}\right)$, at $\mathrm{T}_{1}, \mathrm{~T}_{2}, \mathrm{~T}_{3}$ and $\mathrm{T}_{4}$. The blood samples were collected using a heparinized anticoagulation tube for cytokine concentration analysis.

Cytokine assays. For cytokine measurements, the blood samples were centrifuged at $2,200 \mathrm{xg}$ for $10 \mathrm{~min}$ at $4^{\circ} \mathrm{C}$. The supernatant was stored at $-20^{\circ} \mathrm{C}$ until subsequent use. Plasma concentrations of IL-2 (cat. no. D2050), IL-4 (cat. no. D4050) and IFN- $\gamma$ (cat. no. DIF50C) were detected using ELISA kits (all from R\&D Systems, Inc.) The absorbance was measured at a wavelength of $450 \mathrm{~nm}$ using a Spectra Max 190 microplate reader (Molecular Devices, LLC). To determine the balance of $\mathrm{Th} 1 / \mathrm{Th} 2$, the IL-2/IL-4 ratio was also calculated.

Statistical analysis. Statistical analyses were performed using the SPSS software, (version 19.0; IBM Corp.). Data were tested for

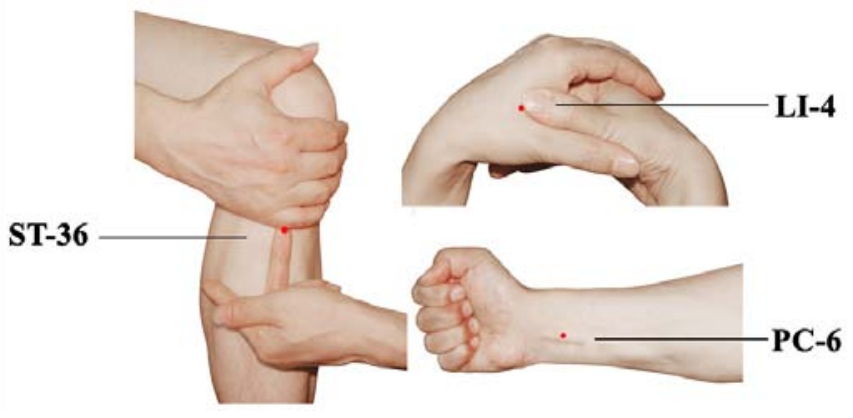

Figure 1. Location of acupoints for TEAS. TEAS, transcutaneous electrical acupoint stimulation.

normality using the Kolmogorov-Smirnov test. Continuous variables are presented as the mean \pm SD. Continuous variables were analyzed using the independent Student's t-test or Mann-Whitney $\mathrm{U}$ test. A mixed two-way ANOVA followed by Bonferroni correction were used to detect comparisons of cytokine concentrations between groups at the same time and within groups at the different time points. Categorical variables are presented as numbers or frequencies. Categorical variables were analyzed using the $\chi^{2}$ test or Fisher's exact test, where appropriate. $\mathrm{P}<0.05$ was considered to indicate a statistically significant difference.

\section{Results}

Participant enrollment. Initially, 80 participants were recruited. Among them, 10 participants (12.50\%) were excluded due to meeting the exclusion criteria and 5 participants $(6.25 \%)$ were excluded for other reasons (Fig. 2). A total of one patient in the TEAS group and two patients in the sham TEAS group were excluded as they refused to receive TEAS after surgery. Similarly, two patients in the TEAS group had not completed all time point stimulations. Therefore, available data from 65 participants $(81.25 \%)$ were included in the analysis.

Demographics and operation details. The demographic characteristics were comparable between the two groups. There were no significant differences between the two groups regarding the details of anesthesia and operation, in terms of duration, operation site, blood loss, infusion volume and urine volume (Table I).

Postoperative analgesia indexes. The postoperative VAS scores at $\mathrm{T}_{2}$ and $\mathrm{T}_{3}$ in the TEAS group were significantly lower compared with the sham TEAS group. There were no statistical differences at $T_{1}$ and $T_{4}$ between the groups (Fig. 3). Moreover, the total consumption of opioids in PCA pump and cumulative times of rescue analgesia during the 48 -h postoperative period were significantly lower in the TEAS group compared with the sham TEAS group (Table II).

Compared with the sham TEAS group, the incidences of PONV and headache were significantly lower in the TEAS group. No significant differences between the two groups were detected regarding the incidence of pruritis and dizziness (Table II).

Immunological indexes. The baseline levels of IL-2, IL-4 and IFN- $\gamma$ at $\mathrm{T}_{0}$ were similar between the two groups. Compared 


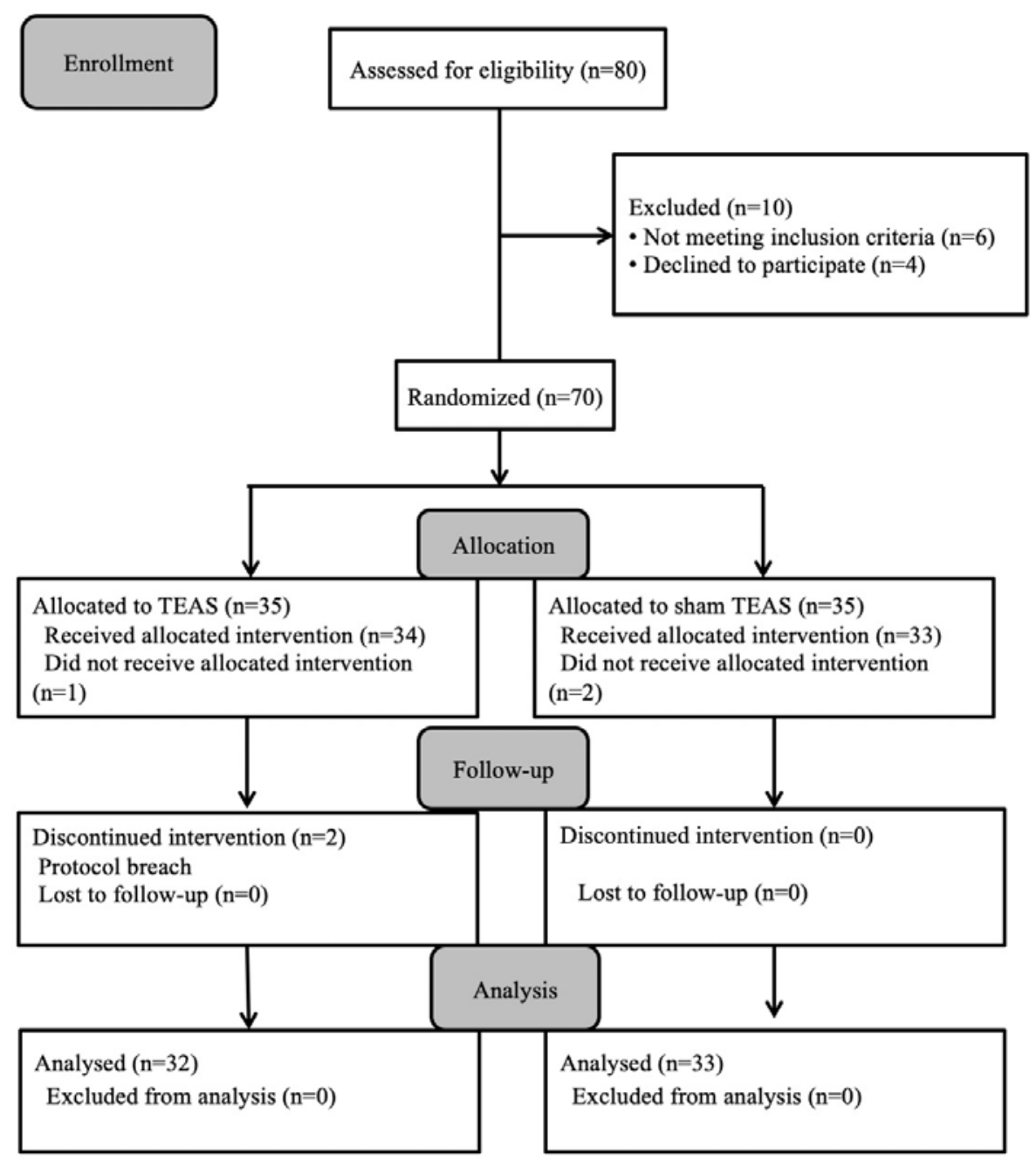

Figure 2. Flow chart showing participants randomized to receive TEAS or Sham TEAS. TEAS, transcutaneous electrical acupoint stimulation.

with baseline levels at $\mathrm{T}_{0}$, serum levels of IL-2, IFN- $\gamma$ and the ratio of IL-2/IL-4 were significantly decreased at $\mathrm{T}_{1}-\mathrm{T}_{4}$ in the sham TEAS group, whereas the serum levels of IL-4 were significantly increased at all postoperative time points. Similarly, in the TEAS group, significantly lower serum levels of IL-2 and IFN- $\gamma$, a decreased IL-2/IL-4 ratio, and higher levels of IL- 4 were observed at $T_{1}, T_{2}$ and $T_{3}$ compared with $T_{0}$. However, postoperative serum levels of IL-2 and IL-4, and the IL-2/IL-4 ratio at $\mathrm{T}_{4}$ were similar to those at $\mathrm{T}_{0}$ in the TEAS group, and the serum levels of IFN- $\gamma$ were still decreased at $\mathrm{T}_{4}$.

In the TEAS group, the serum levels of IL-2 at $\mathrm{T}_{2}-\mathrm{T}_{4}$ and IFN- $\gamma$ at $T_{3}$ and $T_{4}$ were higher compared with the sham TEAS group. By contrast, the serum levels of IL-4 were lower at $\mathrm{T}_{2}-\mathrm{T}_{4}$. Moreover, the IL-2/IL-4 ratio in the TEAS group was significantly higher compared with the sham TEAS group at $\mathrm{T}_{2}-\mathrm{T}_{4}$ (Fig. 4).

\section{Discussion}

Increasing evidence suggested that surgical trauma-induced stress or general anesthesia could lead to immunosuppression (3-5,32). A recent prospective and randomized pilot analysis investigated the effect of anesthetic technique on immunocyte infiltration in breast cancer (33). The analysis indicated that balancing general anesthesia with opioid analgesia could attenuate perioperative immunity. A previous study also indicated that regional anesthesia and avoidance of opioids could reduce perioperative residual disease (34), which was consistent with another study (35).

Postoperative pain could lead to the release of inflammatory factors and result in immune disorder (9). Therefore, minimizing the immunosuppressive regimen has been beneficial for patients with cancer (34-36). Opioids are recommended to relieve postoperative or cancer-related pain $(10,37)$. However, intractable opioid-induced adverse reactions, especially immunosuppression, could be easily overlooked (38). Although weak opioids (for example, tramadol) and non-steroidal anti-inflammatory drugs cannot inhibit immune function $(39,40)$, they are rarely used alone for postoperative pain control, given their weak analgesic effects and side effects. In the present study, TEAS in combination with low-dose opioids was selected for analgesia. The results suggested that patients in the TEAS group displayed significantly lower VAS scores at 12 and $24 \mathrm{~h}$ post-operation, as well as reduced consumption of PCA within $48 \mathrm{~h}$ post-surgery, indicating that TEAS displayed potent analgesic effects, which was consistent with a previous study (27). Therefore, TEAS might be associated with effective postoperative pain relief 
Table I. Clinical characteristics of patients between two groups.

\begin{tabular}{lccc}
\hline Variable & TEAS & Sham TEAS & P-value \\
\hline Average age (years) & $45.6 \pm 9.8$ & $46.9 \pm 8.6$ & 0.572 \\
BMI $\left(\mathrm{kg} / \mathrm{m}^{2}\right)$ & $23.4 \pm 4.2$ & & \\
ASA physical status & & $22.6 \pm 3.3$ & 0.396 \\
I & 26 & 9 & \\
II & 6 & 16 & \\
Operation site & & 17 & 0.426 \\
Left & 18 & $128.6 \pm 25.6$ & $96.1 \pm 20.2$ \\
Right & 14 & $105.6 \pm 25.7$ \\
Anesthesia duration (min) & $131.6 \pm 21.2$ & $910.7 \pm 143.6$ & 0.609 \\
Operation duration (min) & $98.8 \pm 16.8$ & $158.6 \pm 30.1$ \\
Blood loss volume (ml) & $109.5 \pm 28.6$ & 0.561 \\
Infusion volume (ml) & $895.2 \pm 120.5$ & 0565 \\
Urine volume (ml) & $170.3 \pm 25.4$ & 0.636 \\
\hline
\end{tabular}

Values are presented as the mean \pm standard deviation or count. ASA, American Society of Anesthesiologists; TEAS, transcutaneous electrical acupoint stimulation.

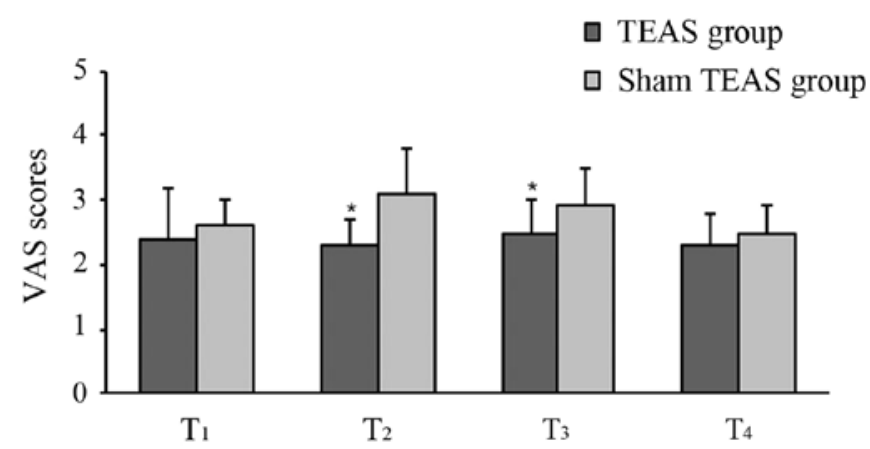

Figure 3. Comparison of VAS scores between two groups. Values are presented as the mean \pm standard deviation. ${ }^{*} \mathrm{P}<0.05$ vs. Sham TEAS group. VAS, Visual Analog Scale; TEAS, transcutaneous electrical acupoint stimulation.

and lower analgesic consumption. In addition, the incidences of nausea, vomiting and headache were lower in the TEAS group, which suggested that TEAS could attenuate the incidence of adverse effects. TEAS displayed an analgesic role via certain intensity stimulation on specific acupoints, which was characterized by low stimulation, strong operability and few side effects. In a previous trial, the therapeutic advantage of pretreatment with TEAS at Jia ji EX-B2 was assessed, and the results indicated reduced post-procedural discomfort and abdominal pain following colonoscopy (41). A meta-analysis including a total of 1,350 participants demonstrated that the use of TENS could significantly reduce the consumption of postoperative analgesics (42), whereas TEAS combined the effect of TENS and acupuncture. In addition, Liang et al (43) reported that TEAS was an effective adjunct to opioid therapy for moderate and severe cancer-related pain, and could reduce side effects and improve immune function. Therefore, the utilization of TEAS may have a direct clinical application value, especially for patients with cancer. In a previous study, the PC6 acupoint stimulation effectively prevented and relieved nausea and vomiting that was associated with surgery (44). In addition, two previously published randomized controlled trials reported that acupuncture at PC6 was associated with the relief of postoperative nausea and vomiting $(45,46)$. ST36 and LI-4 are the most common acupoints for analgesia, and stimulation at LI-4 is especially effective for head and neck pain (47). A previous study indicated that acupuncture at LI-4 was as effective as analgesics in relieving headaches with fewer side effects, which was suggested as an alternative to the non-pharmacological analgesia method (48). In an animal experiment, electroacupuncture (EA) at LI-4 and ST-36 significantly enhanced the immune function of rats with gastric carcinoma after operation (49). Moreover, EA at acupoint ST36 alone in mice may ameliorate inflammation and modulate immune function in collagen-induced arthritis (50). EA at acupoint ST36 has been revealed to be beneficial for preserving immune function and improving postoperative recovery $(17,29)$. Consequently, PC-6, LI-4 and ST-36 acupoints were investigated in the present study.

Although TEAS is widely used in daily clinical practice, the specific analgesic mechanism is not fully understood. TEAS serves as an alternative to acupuncture, which provides stimulation by needles on the surface of target acupoints. TEAS can also generate an action potential to activate nerve fibers $(51,52)$ as signals are transmitted to the central nervous system to induce corresponding analgesic effects by modulating the release of neurotransmitters, such as endorphins and enkephalins, and blocking pain signaling pathways (53). EA has also been reported to slowly increase pain thresholds, producing analgesia via counter-regulation of glial activation $(54,55)$. Furthermore, a neuromodulator with antinociceptive properties, adenosine, has been implicated in mediating the analgesia effect of EA, which could be prolonged by manipulation of adenosine metabolism (56).

The present study demonstrated that immune function in both groups was suppressed after surgery, which was associated 
Table II. Effective press, consumption of opioids and incidence of adverse events.

\begin{tabular}{lcrr}
\hline Variable & TEAS & Sham TEAS & P-value \\
\hline Number of effective press & $6.2 \pm 3.7^{\mathrm{a}}$ & $12.3 \pm 4.6$ & $<0.001$ \\
Consumption of opioids (ml) & $102.8 \pm 7.4^{\mathrm{a}}$ & $120.6 \pm 9.2$ & $<0.001$ \\
Dizziness & 7 & 9 & 0.614 \\
Pruritis & 2 & 4 & 0.672 \\
Headache & $2^{\mathrm{a}}$ & 9 & 0.044 \\
PONV & $4^{\mathrm{a}}$ & 12 & 0.042
\end{tabular}

Values are presented as the mean \pm standard deviation or count. ${ }^{a} \mathrm{P}<0.05$ vs. Sham TEAS group. VAS, Visual Analog Scale; PCA, patient-controlled analgesia; PONV, postoperative nausea and vomiting.

A
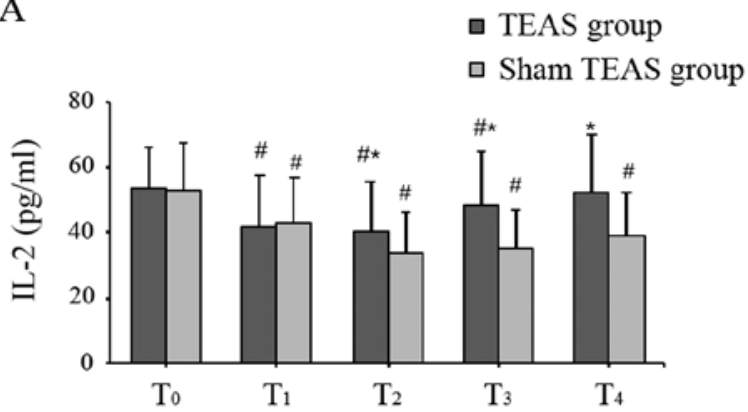

$\mathrm{C}$

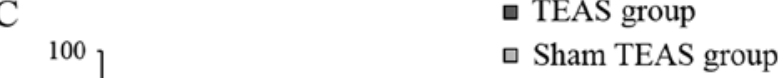

B

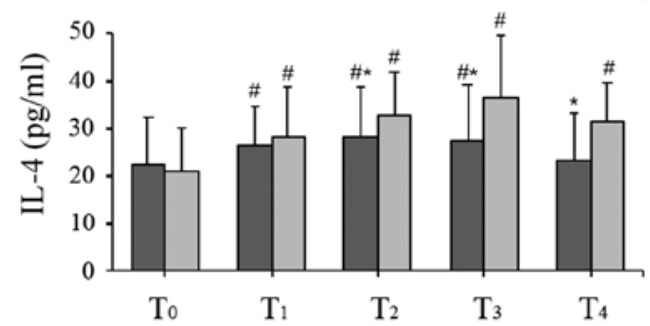

$\mathrm{D}$

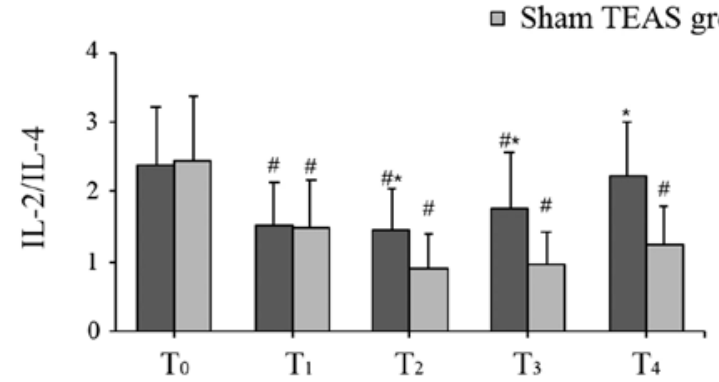

Figure 4. Serum levels of (A) IL-2, (B) IL-4, (C) IFN- $\gamma$ and (D) IL-2/IL-4 ratio between two groups. Values are presented as the mean \pm standard deviation. ${ }^{*} \mathrm{P}<0.05$ vs. sham TEAS group; ${ }^{\#} \mathrm{P}<0.05$ vs. T0. TEAS, transcutaneous electrical acupoint stimulation.

with downregulated serum levels of IL-2 and IFN- $\gamma$, and increased serum levels of IL-4. In the TEAS group, the levels of IL-2 and IL-4 were restored to the preoperative level at $48 \mathrm{~h}$ post-surgery. However, the levels of IL-2 and IL-4 in the sham TEAS group did not restore to the baseline level until $48 \mathrm{~h}$ post-surgery. Moreover, the serum levels of IL-2 at $\mathrm{T}_{2}, \mathrm{~T}_{3}$ and $\mathrm{T}_{4}$, and IFN- $\gamma$ at $\mathrm{T}_{3}$ and $\mathrm{T}_{4}$ in the sham TEAS group were lower compared with the TEAS group. By contrast, the serum levels of IL-4 were higher in the sham TEAS group at $\mathrm{T}_{2}, \mathrm{~T}_{3}$ and $\mathrm{T}_{4}$. TEAS returned the aforementioned cellular immune factors to the preoperative control value at a faster rate, exerting a modulatory effect on the immune system. A previous animal study indicated that EA at the ST36 acupoint significantly enhanced the levels of IFN- $\gamma$ and IL-2 (57). Similarly, surgical trauma has contributed to postoperative immunosuppression, which is associated with decreased expression levels of IL-2 and IFN- $\gamma$, and increased expression of IL-4 in a surgical traumatized rat model (58). In the present study, TAES treatment increased the serum levels of IL-2, IFN- $\gamma$, and decreased IL-4 secretion, suggesting that TAES could attenuate the postoperative immune dysfunction of patients with breast cancer via altering the expression of Th1/Th2 cell-associated cytokines. The results were consistent with a previous study (59). Moreover, Li et al (60) demonstrated that EA combined with anesthesia was able to reduce perioperative immunosuppression compared with general anesthesia alone. Under normal conditions, Th1 and Th2 cells are in a relatively balanced state. Since the shift from Th1 to Th2 is associated with immunosuppression and cancer development, the balance between Th1 and Th2 cells is of importance in patients with cancer (61). The ratio of IL-2/IL-4 is typically used to represent the Th1/Th2 cell ratio $(32,62)$. In the present study, the balance between Th1/Th2 was measured to investigate the impact of EA on immune function. The results suggested that the IL-2/IL-4 ratio was significantly altered secondary to mastectomy, whereas TEAS partially restored the imbalance of Th1/Th2.

Although the exact mechanisms underlying EA stimulation-mediated promotion of the functions of the immune 
system are not completely understood, previous studies have indicated that certain signaling pathways could be associated with the signaling mechanisms $(57,58,63)$. EA administration could regulate the production of Th1 and Th 2 cytokines, and the expression of mRNA splenic T cells (58). Additionally, the immunomodulatory effects of EA are likely connected with the MAPK signaling pathway, which serves an important role in the regulation of $\mathrm{T}$ cell activation and cytokine production (58). EA applied to the ST36 acupoint enhanced the level of immune cytokines and cluster of differentiation 4 in spleen cells via transient receptor potential vanilloid (TRPV) channels (57). The aforementioned study also suggested that the activation of TRPV channels was related to $\mathrm{Ca}^{2+}$ influx in spleen cells. Moreover, by acupuncture application, an increase in neurotransmitter levels, such as $\beta$-endorphin, serotonin, met-encephalin and leu-encephalin have been detected in the central nervous system and plasma, which have been revealed to exhibit immunomodulator effects on the immune system $(63,64)$. However, pain relief could also have the potential to benefit immune function by reducing postoperative stress and inhibiting excessive release of inflammatory cytokines $(9,36)$.

The present study had a number of limitations. Firstly, the present study was a single center study with a strictly defined participant population; therefore, the findings might not be applicable to other centers despite high homogeneity of both groups. Secondly, although the sham group was treated in the same manner as the TEAS group, other than the stimulation, blinding of TEAS treatment was not possible as patients eventually knew whether they were receiving electrical stimulation. Thirdly, it was not possible for the operators to be blinded to the grouping because the effectiveness of TEAS was determined via the sensation of de qi. In addition, the present study did not detect the influences on long-term sequelae, such as tumor metastasis, recurrence and mortality, which could be associated with perioperative immunosuppression. Moreover, the relatively small sample size of the present study may have partially affected the outcomes. Therefore, further studies with larger sample sizes and multi-indicators are required to evaluate the potential advantages of TEAS.

To conclude, the present study indicated that TEAS maintained cellular immune function, alleviated postoperative pain and reduced the occurrence of opioid-related side effects, providing a novel insight for selection of postoperative analgesia. The results of the present study may have implications for postoperative pain management in patients with cancer regarding immune function and postoperative recovery.

\section{Acknowledgements}

Not applicable.

\section{Funding}

No funding was received.

\section{Availability of data and materials}

The datasets used and/or analyzed during the current study are available from the corresponding author on reasonable request.

\section{Authors' contributions}

LA and JS drafted the manuscript. JS and JG were in charge of patient recruitment. LA collected individual data. SZ and YB performed statistical analyses. JG and YB contributed to study conception. JG reviewed the manuscript and the approved final submission. All authors read and approved the final manuscript.

\section{Ethical approval and consent to participate}

The present study was approved by the Ethics Committee of Human Research of Tangshan People's Hospital (approva 1 no. RMYY-YWLL-2017-1110; Tangshan, China). The trial was also registered prior to participant enrollment at www. chictr.org.cn (clinical trial no. ChiCTR1800017768). Written informed consent was obtained from each patient.

\section{Patient consent for publication}

Not applicable.

\section{Competing interests}

The authors declare that they have no competing interests.

\section{References}

1. Torre LA, Siegel RL, Ward EM and Jemal A: Global cancer incidence and mortality rates and trends-an update. Cancer Epidemiol Biomarkers Prev 25: 16-27, 2016.

2. Kurosawa S: Anesthesia in patients with cancer disorders. Curr Opin Anaesthesiol 25: 376-384, 2012.

3. Neeman E and Ben-Eliyahu S: The perioperative period and promotion of cancer metastasis: New outlooks on mediating mechanisms and immune involvement. Brain Behav Immun 30 (Suppl): S32-S40, 2013

4. Green JS and Tsui BC: Impact of anesthesia for cancer surgery: Continuing professional development. Can J Anaesth 60: 1248-1269, 2013.

5. Boland JW, McWilliams K, Ahmedzai SH and Pockley AG: Effects of opioids on immunologic parameters that are relevant to anti-tumour immune potential in patients with cancer: A systematic literature review. Br J Cancer 111: 866-873, 2014.

6. Cho JS, Lee MH, Kim SI, Park S, Park HS, Oh E, Lee JH and Koo BN: The Effects of perioperative anesthesia and analgesia on immune function in patients undergoing breast cancer resection: A prospective randomized study. Int J Med Sci 14: 970-976, 2017.

7. Bobocea AC, Trandafir B, Bolca C and Cordoş I: Minimally invasive surgery in cancer. Immunological response. Chirurgia (Bucur) 107: 154-157, 2012.

8. Leaver HA, Craig SR, Yap PL and Walker WS: Lymphocyte responses following open and minimally invasive thoracic surgery. Eur J Clin Invest 30: 230-238, 2000.

9. Page GG, Blakely WP and Ben-Eliyahu S: Evidence that postoperative pain is a mediator of the tumor-promoting effects of surgery in rats. Pain 90: 191-199, 2001.

10. Rawal N: Current issues in postoperative pain management. Eur J Anaesthesiol 33: 160-171, 2016.

11. Wei G, Moss J and Yuan CS: Opioid-induced immunosuppression: Is it centrally mediated or peripherally mediated? Biochem Pharmacol 65: 1761-1766, 2003.

12. Alonzo NC and Bayer BM: Opioids, immunology, and host defenses of intravenous drug abusers. Infect Dis Clin North Am 16: 553-569, 2002.

13. Chiu HY, Hsieh YJ and Tsai PS: Systematic review and meta-analysis of acupuncture to reduce cancer-related pain. Eur J Cancer Care (Engl): Feb 7, 2017 (Epub ahead of print). doi: 10.1111/ecc.12457. 
14. Gavronsky S, Koeniger-Donohue R, Steller J and Hawkins JW: Postoperative pain: Acupuncture versus percutaneous electrical nerve stimulation. Pain Manag Nurs 13: 150-156, 2012.

15. Chernyak GV and Sessler DI: Perioperative acupuncture and related techniques. Anesthesiology 102: 1031-1049, 2005.

16. Yao Y, Zhao Q, Gong C, Wu Y, Chen Y, Qiu L, Wu X and Chen Y: Transcutaneous electrical Acupoint stimulation improves the postoperative quality of recovery and analgesia after gynecological laparoscopic surgery: A randomized controlled trial. Evid Based Complement Alternat Med 2015: 324360, 2015.

17. Grech D, Li Z, Morcillo P, Kalyoussef E, Kim DD, Bekker A and Ulloa L: Intraoperative low-frequency electroacupuncture under general anesthesia improves postoperative recovery in a randomized trial. J Acupunct Meridian Stud 9: 234-241, 2016.

18. Enwere EK, Kornaga EN, Dean M, Koulis TA, Phan T, Kalantarian M, Köbel M, Ghatage P, Magliocco AM, Lees-Miller SP and Doll CM: Expression of PD-L1 and presence of CD8-positive T cells in pre-treatment specimens of locally advanced cervical cancer. Mod Pathol 30: 577-586, 2017.

19. Schreiber RD, Old LJ and Smyth MJ: Cancer immunoediting: Integrating immunity's roles in cancer suppression and promotion. Science 331: 1565-1570, 2011.

20. Bradley LM,Dalton DK and Croft M: A direct role for IFN-gamma in regulation of Th1 cell development. J Immunol 157: 1350-1358, 1996.

21. Liao W, Lin JX and Leonard WJ: IL-2 family cytokines: New insights into the complex roles of IL-2 as a broad regulator of $\mathrm{T}$ helper cell differentiation. Curr Opin Immunol 23: 598-604, 2011.

22. Ellyard JI, Simson L and Parish CR: Th2-mediated anti-tumour immunity: Friend or foe? Tissue Antigens 70: 1-11, 2007.

23. Han YF, Zhao J, Ma LY, Yin JH, Chang WJ, Zhang HW and Cao GW: Factors predicting occurrence and prognosis of hepatitis-B-virus-related hepatocellular carcinoma. World J Gastroenterol 17: 4258-4270, 2011.

24. International Conference on Harmonization of Technical Requirements for Registration of Pharmaceuticals for Human Use. ICH Harmonized Tripartite Guideline. [Accessed May 12, 2015]; Guideline for Good Clinical Practice E6(R1) 1996 Available from: http://www.ich.org/fileadmin/Public_Web_Site/ICH_ Products/Guidelines/Efficacy/E6/E6_R1_Guideline.pdf.

25. American Society of Anesthesiologists: ASA physical status classification system. [Accessed 23 Nov 2018] Available from: https://www. asahq.org/resources/ American Society of Anesthesiologists.

26. Huang S, Peng W, Tian X, Liang H, Jia Z, Lo T, He M and Feng Y: Effects of transcutaneous electrical acupoint stimulation at different frequencies on perioperative anesthetic dosage, recovery, complications, and prognosis in video-assisted thoracic surgical lobectomy: A randomized, double-blinded, placebo-controlled trial. J Anesth 31: 58-65, 2017.

27. Tu Q, Gan J, Shi J, Yu H, He S and Zhang J: Effect of transcutaneous electrical acupoint stimulation on postoperative analgesia after ureteroscopic lithotripsy: A randomized controlled trial. Urolithiasis 47: 279-287, 2019.

28. Qu F, Li R, Sun W, Lin G, Zhang R, Yang J, Tian L, Xing GG, Jiang $\mathrm{H}$, Gong F, et al: Use of electroacupuncture and transcutaneous electrical acupoint stimulation in reproductive medicine: A group consensus. J Zhejiang Univ Sci B 18 186-193, 2017

29. Tu Q, Yang Z, Gan J, Zhang J, Que B, Song Q and Wang Y: Transcutaneous electrical acupoint stimulation improves immunological function during the perioperative period in patients with non-small cell lung cancer undergoing video-assisted thoracic surgical lobectomy. Technol Cancer Res Treat: Jan 1, 2018 (Epub ahead of print). doi: 10.1177/1533033818806477.

30. Oliveira CR, Bernardo WM and Nunes VM: Benefit of general anesthesia monitored by bispectral index compared with monitoring guided only by clinical parameters. Systematic review and meta-analysis. Braz J Anesthesiol 67: 72-84, 2017.

31. Huskisson E: Measurement of pain. Lancet 2: 1127-1131, 1974.

32. Cata JP, Bauer M, Sokari T, Ramirez MF, Mason D, Plautz G and Kurz A: Effects of surgery, general anesthesia, and perioperative epidural analgesia on the immune function of patients with non-small cell lung cancer. J Clin Anesth 25: 255-262, 2013.

33. Fiona Desmond J, McCormack N, Mulligan M, Stokes and Donal JB: Effect of anesthetic technique on immune cell infiltrationin breast cancer: A follow-up pilot analysis of a prospective, randomised, investigator-masked study. Anticancer Res 35: 1311-1319, 2015.
34. Buckley A, McQuaid S, Johnson P and Buggy DJ: Effect of anaesthetic technique on the natural killer cell anti-tumour activity of serum from women undergoing breast cancer surgery: A pilot study. Br J Anaesth 113 (Suppl 1): i56-i62, 2014.

35. Pérez-González O, Cuéllar-Guzmán LF, Soliz J and Cata JP: Impact of regional anesthesia on recurrence, metastasis, and immune response in breast cancer surgery: A systematic review of the literature. Reg Anesth Pain Med 42: 751-756, 2017.

36. Kim SY, Kim NK, Baik SH, Min BS, Hur H, Lee J, Noh HY, Lee JH and Koo BN: Effects of postoperative pain management on immune function after laparoscopic resection of colorectal cancer: A Randomized Study. Medicine (Baltimore) 95: e3602, 2016.

37. Fallon M, Giusti R, Aielli F, Hoskin P, Rolke R, Sharma M and Ripamonti CI; ESMO Guidelines Committee. Electronic address: Clinicalguidelines@esmo.org: Management of cancer pain in adult patients: ESMO Clinical Practice Guidelines. Ann Oncol 29 (Suppl 4): iv166-iv191, 2018.

38. Suzuki M, Sakurada T, Gotoh K, Watanabe S and Satoh N: Correlation between the administration of morphine or oxycodone and the development of infections in patients with cancer pain. Am J Hosp Palliat Care 30: 712-716, 2013.

39. Sacerdote P, Bianchi M, Gaspani L, Manfredi B, Maucione A, Terno G, Ammatuna M and Panerai AE: The effects of tramadol and morphine on immune responses and pain after surgery in cancer patients. Anesth Analg 90: 1411-1414, 2000.

40. Narahara H, Kadoi Y, Hinohara H, Kunimoto F and Saito S: Comparative effects of flurbiprofen and fentanyl on natural killer cell cytotoxicity, lymphocyte subsets and cytokine concentrations in post-surgical intensive care unit patients: Prospective, randomized study. J Anesth 27: 676-683, 2013.

41. Chen Y,Wu W, Yao Y, Yang Y,Zhao Q and Qiu L: Transcutaneous electric acupoint stimulation at Jiaji points reduce abdominal pain after colonoscopy: A randomized controlled trial. Int J Clin Exp Med 8: 5972-5977, 2015.

42. Bjordal JM, Johnson MI and Ljunggreen AE: Transcutaneous electrical nerve stimulation (TENS) can reduce postoperative analgesic consumption. A meta-analysis with assessment of optimal treatment parameters for postoperative pain. Eur J Pain 7: 181-188, 2003.

43. Liang Y, Bao G, Gong L, Zhou J, Kong X, Ran R, Shao X, Jiang Y, Zhang W, Liu B, et al: Evaluating the analgesic effect and advantage of transcutaneous electrical acupoint stimulation combined with opioid drugs for moderate to severe cancer-related pain: A study protocol for a randomized controlled trial. Trials 20: 40,2019.

44. Lee A and Fan LT: Stimulation of the wrist acupuncture point P6 for preventing postoperative nausea and vomiting. Cochrane Database Syst Rev: April 15, 2009 (Epub ahead of print). doi: 10.1002/14651858.CD003281.pub.

45. Cooke M, Rickard C, Rapchuk I, Shekar K, Marshall AP, Comans T, Doi S, McDonald J and Spooner A: PC6 acupoint stimulation for the prevention of postcardiac surgery nausea and vomiting: A protocol for a two-group, parallel, superiority randomised clinical trial. BMJ Open 4: e006179, 2014.

46. Lee S, Lee MS, Choi DH and Lee SK: Electroacupuncture on PC6 prevents opioid-induced nausea and vomiting after laparoscopic surgery. Chin J Integr Med 19: 277-281, 2013.

47. Shen YF and Goddard G: The short-term effects of acupuncture on myofascial pain patients after clenching. Pain Pract 7: 256-264, 2007.

48. Witt CM, Reinhold T, Jena S, Brinkhaus B and Willich SN: Cost-effectiveness of acupuncture treatment in patients with headache. Cephalalgia 28: 334-345, 2008

49. Lai M, Wang SM, Wang Y, Tang CL, Kong LW and Xu XY: Effects of electroacupuncture of 'Zusanli' (ST 36), 'Hegu' (LI 4) and/or 'Sanyinjiao' (SP 9) on immunofunction in gastric carcinectomy rats. Zhen Ci Yan Jiu 33: 245-249, 2008 (In Chinese).

50. Yim YK, Lee H, Hong KE, Kim YI, Lee BR, Son CG and Kim JE: Electro-acupuncture at acupoint ST36 reduces inflammation and regulates immune activity in Collagen-Induced Arthritic Mice. Evid Based Complement Alternat Med 4: 51-57, 2007.

51. Kagitani F, Uchida S, Hotta $\mathrm{H}$ and Aikawa Y: Manual acupuncture needle stimulation of the rat hindlimb activates groups I, II, III and IV single afferent nerve fibers in the dorsal spinal roots. Jpn J Physiol 55: 149-155, 2005.

52. Han JS: Acupuncture: Neuropeptide release produced by electrical stimulation of different frequencies. Trends Neurosci 26: 17-22, 2003.

53. Acar HV: Acupuncture and related techniques during perioperative period: A literature review. Complement Ther Med 29: 48-55, 2016. 
54. Zhao ZQ: Neural mechanism underlying acupuncture analgesia Prog. Neurobiol 85: 355-375, 2008.

55. Lin L, Skakavac N, Lin X, Lin D, Borlongan MC, Borlongan CV and Cao C: Acupuncture-induced analgesia: The role of microglial inhibition. Cell Transplant 25: 621-628, 2016.

56. Goldman N, Chen M, Fujita T, Xu Q, Peng W, Liu W, Jensen TK, Pei Y, Wang F, Han X, et al: Adenosine A1 receptors mediate local anti-nociceptive effects of acupuncture. Nat Neurosci 13: 883-888, 2010.

57. Chen L, Xu A, Yin N, Zhao M, Wang Z, Chen T, Gao Y and Chen Z: Enhancement of immune cytokines and splenic $\mathrm{CD}^{+}$ $\mathrm{T}$ cells by electroacupuncture at ST36 acupoint of SD rats. PLoS One 12: e0175568, 2017.

58. Wang K, Wu H, Wang G, Li M, Zhang Z and Gu G: The effects of electroacupuncture on TH1/TH2 cytokine mRNA expression and mitogen-activated protein kinase signaling pathways in the splenic T cells of traumatized rats. Anesth Analg 109: 1666-1673, 2009.

59. Wu H, Wang K, Li G, Meng D, Han J, Wang G and Li YU: Effects of transcutaneous acupoint electrical stimulation on the imbalance of $\mathrm{Th}_{1}, \mathrm{Th}_{2}, \mathrm{Th}_{17}$ and $\mathrm{T}_{\text {reg }}$ cells following thoracotomy of patients with lung cancer. Exp Ther Med 11: 495-502, 2016.
60. Li G, Li S, Wang B and An L: The effect of electroacupuncture on postoperative immunoinflammatory response in patients undergoing supratentorial craniotomy. Exp Ther Med 6: 699-702, 2013.

61. Hao CJ, Li J, Liu P, Li XL, Hu YQ, Sun JC and Wei Y: Effects of the balance between type 1 and type $2 \mathrm{~T}$ helper cells on ovarian cancer. Genet Mol Res: Jun 3, 2016 (Epub ahead of print). doi: $10.4238 / \mathrm{gmr} .15027936$.

62. Woo JH, Baik HJ, Kim CH, Chung RK, Kim DY, Lee GY and Chun EH: Effect of propofol and desflurane on immune cell populations in breast cancer patients: A randomized trial. J Korean Med Sci 30: 1503-1508, 2015.

63. Su TF, Zhang LH, Peng M, Wu CH, Pan W, Tian B, Shi J, Pan HL and Li M: Cannabinoid CB2 receptors contribute to upregulation of $\beta$-endorphin in inflamed skin tissues by electroacupuncture. Mol Pain 7: 98, 2011.

64. Cabioğlu MT and Cetin BE: Acupuncture and immunomodulation. Am J Chin Med 36: 25-36, 2008.

This work is licensed under a Creative Commons Attribution-NonCommercial-NoDerivatives 4.0 International (CC BY-NC-ND 4.0) License. 